\section{P62 SEX DIFFERENCES IN PRIMARY HEALTH CARE USE BEFORE AND AFTER HOSPITAL ADMISSION FOR ACUTE AND CHRONIC CONDITIONS. A REGISTER-BASED COHORT STUDY OF THE DANISH POPULATION AGED 60+}

\begin{abstract}
${ }^{1,2} \mathrm{~A}$ Höhn*, 'J Gampe, ${ }^{1,3} \mathrm{R}$ Rau, ${ }^{2,4} \mathrm{R}$ Lindahl-Jacobsen, ${ }^{2,4,5} \mathrm{~K}$ Christensen, ${ }^{1} \mathrm{~A}$ Oksuzyan. ${ }^{1}$ Max Planck Institute for Demographic Research, Rostock, Germany; ${ }^{2}$ University of Southern Denmark, Odense, Denmark; ${ }^{3}$ University of Rostock, Rostock, Germany; ${ }^{4}$ Max Planck Odense Center on the Biodemography of Aging, Odense, Denmark; ${ }^{5}$ Danish Aging Research Center, Odense, Denmark
\end{abstract}

\subsection{6/jech-2018-SSMabstracts. 185}

Background Empirical studies have consistently reported that women have a mortality advantage at all ages as well as with respect to most adverse health conditions and stressful events during the life course. In seeking to explain this advantage, the existing literature has pointed towards the effects and the interactions of biological, behavioral, and social factors. Among the non-biological factors, a large body of previous research has shown that men tend to seek medical help later and less than women, which can lead to delays in diagnosis and treatment. In this study, we explore the sex differences in primary health care use, before and after admission to hospital for chronic and acute conditions to explore whether the sex differences in treatment-seeking behavior change when health worsens.

Data This is a population-based, longitudinal study with nationwide coverage of the population alive and residing in Denmark at age $60+$ in 1999 . The study population was identified by linking information from the National Health Service Register, the National Patient Register, and the Central Population Register, using a 5\% random sample of the Danish population. The study population was followed up for hospital admissions within the period 1999-2008, and GP contacts within the period 1996-2011. We used a generalized additive mixed model (GAMM) to account for repeated observations and to allow non-linear trajectories within the observed period, which covers the three years before and after hospital admission.

Results We found women having consistently more GP contacts than men before and after admission to hospital. The sex differentials in GP contacts were consistently lower for chronic than for acute conditions. For chronic conditions, the sex differentials were small in both periods: while they narrowed in the period before, they were not significant in the period after admission to hospital. For acute conditions, the sex differentials were smaller in the period after admission to hospital when compared with the period before admission to hospital.

Conclusion Our study indicates smaller sex differences in primary health care use following health deterioration, pointing towards a narrowing of the sex differences in treatment-seeking behavior as a result of the presence of symptoms and the experience of a worsening of the health status.

\section{P63 WHAT DRIVES MENTAL HEALTH INEQUALITIES IN THE UK? A REVIEW OF THEORY AND THEORY-BASED EVIDENCE}

SL Prady ${ }^{*}$ KE Pickett. Department of Health Sciences, University of York, York, UK

10.1136/jech-2018-SSMabstracts. 186

Rationale Globally, those living in less advantaged circumstances have an increased likelihood of having a common mental disorder such as anxiety or depression, and a decreased likelihood that this disorder will be recognised, diagnosed and effectively treated. This is the mental health inequality, which causes significant unfair morbidity. The theory of fundamental causes posits that social circumstances drive health inequalities. Under this framework, social circumstances determine access to 'resources' such as money, knowledge, and connection to others. These resources are leveraged to avoid risks that lead to illness, or minimise illness. Such strategies are unavailable to people who have limited access to resources. Fundamental causes is well accepted as an overarching framework, but it is deliberately general as specific mechanisms of action will vary over time, setting and circumstance. Poor understanding about specific mechanisms of action, however, limits our ability to design effective interventions. Detailed theories that are compatible with fundamental causes have been described, but not well synthesised.

There is a large observational literature describing mental health inequalities in the UK but it is unknown whether this empirical evidence supports or refutes theoretical mechanisms of action.

Aim The overarching aim is to map theories and theory-based evidence to answer two related questions; why, and how, do social circumstances drive UK mental health inequalities?

Methods An iterative and flexible strategy of searching literature and expert consultation was applied to locate health inequality theories. Theories were included if they were compatible with fundamental causes and explained why social circumstances drive health inequalities in the developed world. Database searching was mainly used to amass observational studies from the UK that described mental health inequality in prevalence, diagnosis or treatment. A causal inference critique will be applied to studies that tested a theory, or used theory to explain results. Theories and associated support will be mapped in a logic model. If feasible, a simplified, supported, model (or models) will be deduced.

Population health relevance Experimental or policy-driven interventions to improve population mental health and reduce inequalities are expensive and time-consuming. The evidence base is consequently sparse. Other than in general terms, e.g. downstream interventions increase inequalities, the use of theory in developing mental health interventions is underutilised. This review will highlight evidence-based plausible mechanisms of action which could be used to inform the design of robust mental health interventions that operate at the maximal point to improve population health while reducing inequalities.

\section{P64 ARE MENTALLY DISORDERED OFFENDERS ADEQUATELY PLACED TOWARDS REGULAR OR FORENSIC CARE SETTINGS ACCORDING TO SECURITY NEEDS? A CROSS SECTIONAL STUDY}

D Bourmorck*, P Nicaise, M Molera-Gui, V Lorant, on behalf of Care Study Group. Institute of Health and Society (IRSS), Universite Catholique de Louvain, Brussels, Belgium

\subsection{6/jech-2018-SSMabstracts. 187}

Rationale Access to mental health care for Mentally Disordered Offenders (MDOs) is a common issue across Western countries. MDOs are people with mental disorders who have committed a criminal offence. Their legal condition adds complexity to the multiple fields already involved in regular 